\title{
Mathematical Model of Fibrin Polymerization
}

\author{
A.I. Lobanov ${ }^{1 *}$, A.V. Nikolaev ${ }^{2}$, T.K. Starozhilova ${ }^{1}$ \\ ${ }^{1}$ Chair of Applied Mathematics, Moscow Institute of Physics and Technology, Moscow, Russia \\ ${ }^{2}$ Goldansky Department, Institute of Chemical Physics RAS, Moscow, Russia
}

\begin{abstract}
Blood clotting system (BCS) modelling is an important issue with a plenty of applications in medicine and biophysics. The BCS main function is to form a localized clot at the site of injury preventing blood loss. Mutual influence of fibrin clot consisting mainly of fibrin polymer gel and blood flow is an important factor for BCS to function properly. The process of fibrin polymer mesh formation has not adequately been described by current mathematical models. That is why it is not possible to define the borders of growing clot and model its interaction with a blood flow. This paper main goal is to propose physically well-founded mathematical model of fibrin polymerization and gelation. The proposed model defines the total length of fibrin polymer fibers in the unit volume, determines a position of the border between gel and liquid and allows to evaluate the permeability of growing gel. Without significant structural changes the proposed model could be modified to include the blood shear rate influence on the fibrin polymerization and gelation.
\end{abstract}

Key words: fibrin, clotting, polymerization, gelation

AMS subject classification: $12 \mathrm{~A} 34,56 \mathrm{~B} 78$

\section{Introduction}

Blood clotting system (BCS) prevents blood loss maintaining blood vessels integrity by localizing site of vascular injury and forming a plug. At the same time more than half deaths worldwide could be attributed to BCS malfunctions such as bleeding or thrombosis as an immediate cause of death $[1,10]$.

${ }^{*}$ Corresponding author. E-mail:alexey.i.lobanov@gmail.com 
There is no complete understanding of all processes and their mutual influence while blood clotting so far. Traditionally cellular and plasma stages of hemostasis are distinguished. Cellular stage leads to formation of a platelet plug around the vascular injury site. Plasma (or secondary) stage leads to formation of fibrin-polymer and its gelation. The fibrin polymer mesh interlinks platelets in plugs and makes clot mechanically stable and virtually hermetical. From the biochemical point of view the secondary stage is quite well studied — probably the majority of reactions with their rates and conditions are known. Also some mathematical models of process are built with a various degree of detalization $[22,13,2,3,4]$. There are more than 30 components participating in secondary hemostasis reactions, with some of the latter forming positive and negative feedback loops. Due to heterophase nature of BCS plasma stage and significant role of heterogeneity in component distribution it is hard to predict not only a behavior of the system in a whole but also the influence of some parameters $[22,13]$.

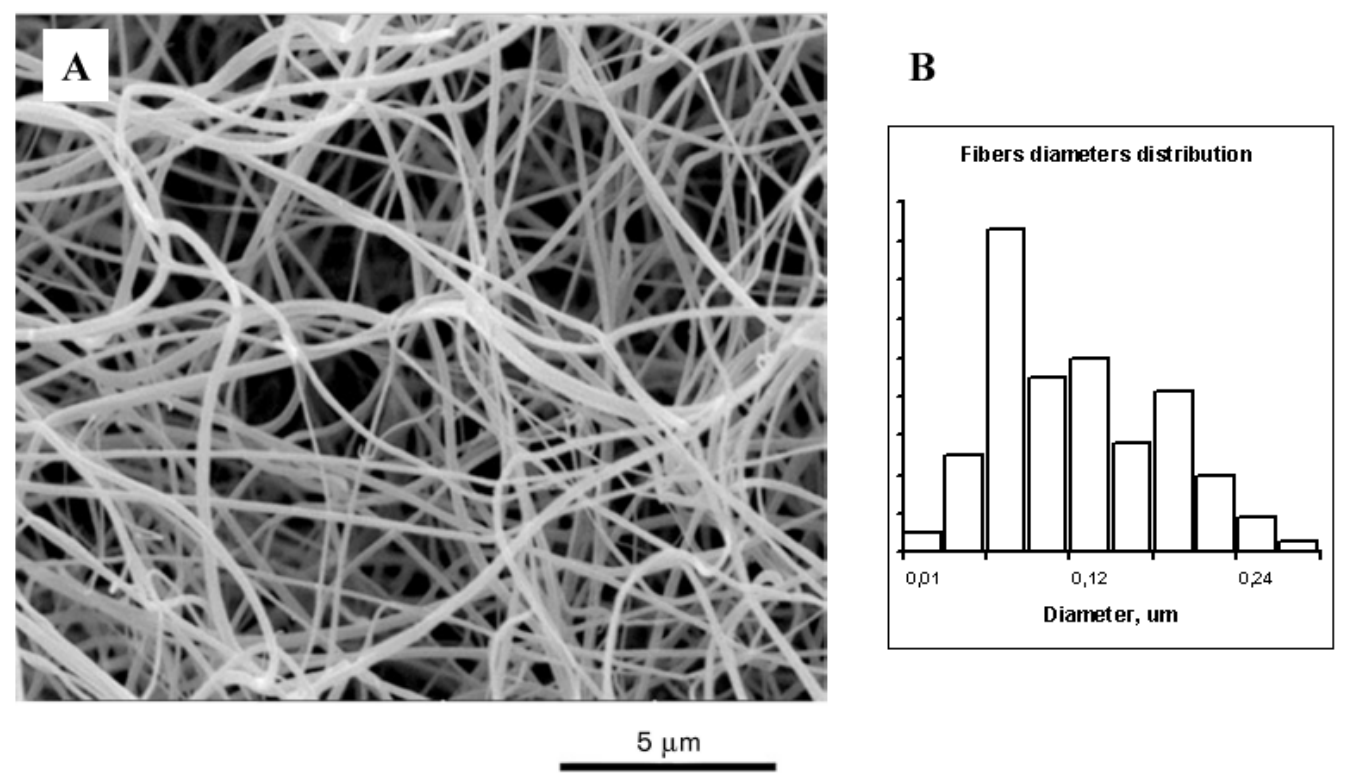

Figure 1: A - scanning electron micrography (SEM) of fibrin-polymer [17]. Fibrinogen from healthy donor plasma has been used for this experiment. Purified fibrinogen in concentration of $0,2 \mathrm{mg} / \mathrm{ml}$ has been polymerized in reconstructed system after thrombin addition $(0,2 \mathrm{NIH} \mathrm{U} / \mathrm{ml})$. $\mathrm{B}$ - SEM image fibers diameters histogram A.

The main goal of this paper is to propose physically well-founded mathematical model of fibrin polymerization and gelation on the basis of fibrin and fibrin-polymer properties.

As the result of reaction operation the cascades of plasma stage thrombin are generated. The fibrin polymerization is initiated by thrombin via the enzymatic cleavage of the fibrinopeptides, converting fibrinogen to fibrin. In turn, thrombin is generated as the result of BCS reaction cascades action. On scales up from $0.5 \mu \mathrm{m}$ the fibrin-polymer could be described as a mesh, consisting of branched threads that is fibers (see Fig. 1). This mesh is quite sparse. The following example can 
help to understand it: if in certain plasma volume all fibrinogen are converted into fibrin-polymer, then the total mesh protein mass will not exceed $4 \mathrm{mg} / \mathrm{ml} \mathrm{[26].} \mathrm{If} \mathrm{the} \mathrm{structural} \mathrm{water} \mathrm{in} \mathrm{fibrin}$ polymer are accounted for, then total mesh mass and relative volume could be estimated as 14-18 $\mathrm{mg} / \mathrm{ml}$ and 1.2-1.6, respectively [9]. Fibrin polymer fiber is a spiral paracrystalline structure with axial repeat around $22.5 \mathrm{~nm}$ and spiral step $2 \mu \mathrm{m}$ [25].

Fiber diameter distribution depends on condition of polymerization and fibrin activation $[9,7]$. Under physiological like conditions the fiber diameter is within $60 \div 140 \mathrm{~nm}$, and mesh "pores" are within $0.1 \div 10 \mu \mathrm{m}[11,8]$. Despite of such "thin build" fibrin polymer mesh is virtually non-permeable for fluid. Darcy permeability of fibrin gel is of the same magnitude order as a clay permeability [11].

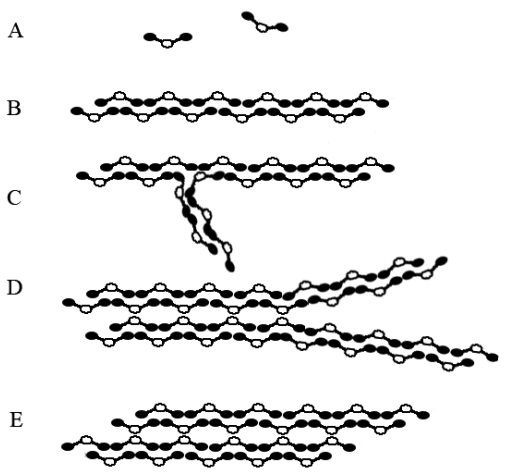

Figure 2: Fibrin forms while sol stage of polymerization. (A) Fibrin monomers in solution. (B) Double stranded fibril. Monomers in fibril shifted in one-half length $(22.5 \mathrm{~nm})$ one relative to another. (C) Trimolecular branching point seems to appear when monomers polymerize like threepointed star. (D) Ordinary tetramolecular branch. Probably, it appears when two fibrils do not completely aggregate laterally. (E) Laterally aggregated fibrils with a small shift relative to each other.

Until recently the majority of BCS models have included the fibrin polymerization in a very straightforward simplified way [2, 3, 22]. The fibrin-polymer mesh was considered to instantly appear in volume if concentration of monomers overcame the certain threshold level. The threshold level itself was estimated from experimental data. Naturally, the experiments picked for threshold estimation were spent in conditions as close as possible to the model assumptions $[3,5,6]$.

Current biochemical experimental data allow to more or less clearly distinguish three stages in polymerization of fibrin, although these stages reactions could proceed simultaneously [21, 24]:

1. enzymatic cleavage of fibrinogen to fibrin-monomer (fibrin monomer length is approximately $45 \mathrm{~nm}$ );

2. self-assembly of fibrin into organized polymer structure; 
3. "cross-linking" - occurrence of stable covalent bonds between neighbour fibrin monomers in polymer structure under FXIIIa action.

Stage 2 could be divided at least into two different elementary processes: an assembly of linear fibrin oligomers of $12 \div 20$ monomers in size and oligomer-oligomer interaction. Sometimes oligomers may have a three-pointed star shape [20]. Oligomers linear parts have a half-staggered double-stranded structure with a period of $22.5 \mathrm{~nm}$ which is a half length of monomer (see Fig. 2) [24]. Experiments proved an existence of the lateral aggregation process between oligomer the oligomers and oligomers with larger structures called fibers. Either an existence of non-complete lateral aggregation resulting in formation of Y-like forks or an interaction leading to a fiber crossings formation can also be supposed.

Unlike polymerization on "molecular level", or evolution of fibrin(ogen) molecule itself, the polymerization on "aggregate level" and gelation process has been studied much worse. Merely a presence of the polymerization stages makes their analysis and modelling a tricky business., As it has been pointed above, some reactions also run in a concurrent manner and system could have states where fibrinogen and fibrin do coexist on different polymerization stages. From the polymer science point view fibrin gelation is strong, as bounds between monomers, oligomers and aggregates are stable [14].

Summarizing different sources data, it can be assumed that under physiological conditions the qualitative description of fibrin polymerization with sol-gel transition should look like:

1. fibrinogen to monomer fibrin conversion by thrombin;

2. polymerization of fibrin monomers to protofibrils, i.e. linear and branched oligomers with 12 to 30 monomer units in size;

3. aggregation;

(a) lateral aggregation of protofibrils with more than certain threshold level length $(N>$ 12) into thin fibers which diameter is approximately equal to doubled diameter of protofibril. Possibly with longitudinal shift and elongation of the resulting aggregate;

(b) crossing of protofibrils and thin fibers with resulting formation of complicated aggregates;

4. polymer scaffold formation and gelation itself. Presumably, on this stage $2 / 3$ of all branching points have already been formed;

5. scaffold thickening, and its enlargement on the gel border by new protofibrils or thin fibers forming from inside or "falling" on the border from outside of the gel due to advection or diffusion;

6. partial rearrangement of polymer, sticking together of some thin fibers forming fibre bundles.

It is worth to note, that processes related to stages 1 and 2 could continue constantly. Stages $3 \mathrm{a}$ and $3 \mathrm{~b}$ are essentially competitive and run concurrently. Conditions and kinetic properties of the stages 1 and 2 could lead to a domination of either $3 \mathrm{a}$ or $3 \mathrm{~b}$ [16]. It is also apparent that 
after the stage 4 in the gelated volume the process 3 will either significantly slow down or extinct at all. Instead, processes 5 and 6 will start as newly formed oligomers will readily interact with surrounding gel scaffold on its border. The significant detail of this scenario is that the fibrin monomers are expected to attach to free oligomers, not to oligomer scaffold, albeit the latter is not explicitly prohibited. Notably, in the above mentioned scenario the polymerization and gelation processes will significantly depend on shear rates of surrounding and nearby fluid flows.

In conclusion it should be noted, that the fibrin-polymer mesh formation mathematical model has a significant applied value. Such model should consider a possibility of intermediate polymerization products transfer, a forming mesh influence on the flow and a shear flow impact on polymerization and gelation itself.

\section{Mathematical model}

\subsection{Fibrin polymerization process}

There are two especially interesting questions in the description of polymerization process: gel nucleus formation and gel volume growth dynamics. Detailed study of polymerization process chemical reactions makes it possible to associate the above mentioned processes within one closed mathematical model.

Semi dilution of oligomer solution can be chosen as criteria of gel nucleus formation. It is considered that at the point, when oligomer concentration achieves some threshold value, the fast oligomers aggregation takes place with a formation of fibrin-polymer gel threads. As a main characteristic of such a thread its length in the unit of volume can be taken.

Let $c_{i}$ is a concentration of oligomers with $i$ monomer units. Let's assume that production of such oligomer (in particular $c_{12}$ ) is a chain polymerization process. Authors offer to use the oligomer $c_{12}$ as a basic component for lateral aggregation and gelation process reactions. That is why chain polymerization reactions that lead to formation of oligomers larger than $c_{12}$ are dropped. Keeping that in mind let us write:

$$
\begin{aligned}
& \frac{\partial c_{1}}{\partial t}=D_{1} \Delta c_{1}+k_{f} c_{\mathrm{fg}} c_{I I a}-2 k_{\text {poly }} c_{1} c_{1}-k_{\text {poly }} c_{1}\left(\sum_{i=2}^{11} c_{i}\right) \\
& \frac{\partial c_{2}}{\partial t}=D_{2} \Delta c_{2}+k_{\text {poly }} c_{1} c_{1}-k_{\text {poly }} c_{2} c_{1}-2 k_{\text {poly }} c_{2} c_{2} \\
& \frac{\partial c_{3}}{\partial t}=D_{3} \Delta c_{3}+k_{\text {poly }} c_{2} c_{1}-k_{\text {poly }} c_{3} c_{1} \\
& \frac{\partial c_{4}}{\partial t}=D_{4} \Delta c_{4}+k_{\text {poly }} c_{3} c_{1}-k_{\text {poly }} c_{4} c_{1}+k_{\text {poly }} c_{2} c_{2}
\end{aligned}
$$




$$
\begin{aligned}
& \frac{\partial c_{5}}{\partial t}=D_{5} \Delta c_{5}+k_{\text {poly }} c_{4} c_{1}-k_{\text {poly }} c_{5} c_{1}, \\
& \cdots \\
& \frac{\partial c_{11}}{\partial t}=D_{11} \Delta c_{11}+k_{\text {poly }} c_{10} c_{1}-k_{\text {poy }} c_{11} c_{1},
\end{aligned}
$$

where $k_{\text {poly }}$ is the rate constant of polymerization reaction, $c_{f g}$ is the fibrinogen concentration, $c_{I I a}$ is the thrombin concentration, $c_{1}$ is the fibrin monomer concentration, $c_{2}, \ldots, c_{12}$ are the concentrations of oligomers with size from 2 to 12 monomers, $D_{1}, \ldots, D_{12}$ are the diffusion coefficients for fibrin oligomers.

The equations shown above describe the process n-unit oligomer production from $(n-1)$ th by fibrin monomer attachment. The speed constant for polymerization reaction of $\mathrm{n}$-th oligomer and fibrin monomer is known to be independent of oligomer size [15]. Also model neglects mutual interaction of oligomers with length above 2. Of course, reactions between large oligomers should proceed. But according to such oligomers molecular structure data [19, 24], these oligomers are long molecules with a large mass starting from size 3. So, such molecules have very low rotational and translational diffusion coefficients. To join the formation of linear oligomer such molecules should not only meet but should also have a proper orientation toward each other. Due to the structural features a fibrin monomer in solution can have different conformations ranging from rod to sphere [24]. Dimer is considerably larger, but also has possibility to "fold" in a conformation which could be described as an elliptical body with eccentricity ratio around 0.5. Trimers and oligomers of a greater size will certainly have one or more completely stretched fibrin monomer. Thus, their shape could be described properly as a rod like. Coefficients of rotational and translational diffusion for such oligomers under rigid rod assumption [12] can be described as:

$$
D_{i}=\frac{\ln \left(L_{i} / b_{i}\right)}{3 \pi \eta L_{i}} k_{B} T, \quad D_{r i}=\frac{3 \ln \left(L_{i} / b_{i}-\varsigma\right)}{\pi \eta L_{i}^{3}} k_{B} T, \quad \varsigma \approx 0.8
$$

where $\eta$ is a dynamical viscosity of solvent, $b_{i}$ is small diameter of fibrin oligomer $\left(b_{i}=2 b_{1}\right.$ for $i>1$ ), $L_{i}$ is length of fibrin oligomer (for $i>1, L_{i}=(i+1) L_{1} / 2$, e.g. $L_{12}=296 \mathrm{~nm}$ ). Apparently, the oligomer size increase results in fast decrease of both coefficients, in turn that will decrease probability of mentioned reactions drastically. So, the first assumption of the model: mutual reactions of oligomers with size larger than 1 could be dropped except for the reaction of two dimmers forming a tetramer, and the speed constant for remained polymerization reactions do not depend on oligomer size.

Currently there are quite few mathematical models which account for thrombin generation and cleavage as well as fibrinogen concentration decay $[3,4,22]$, but this effect is set aside of this model scope. Now we also neglect the fact that fibrinogen activation is itself a complicated multistage process. Any intermediate states of incompletely activated fibrin are not considered. Notably, any reasonable model of thrombin evolution and fibrinogen activation can be connected with the current polymerization description. 
Below we will track concentration $\left(c_{12}\right)$ of oligomer with size of 12 monomer units:

$$
\frac{\partial c_{12}}{\partial t}=D_{12} \Delta c_{12}+k_{\text {poly }} c_{11} c_{1}
$$

Second term in the right part of the equation describes production of oligomer as a result of oligomer 11 and monomeric fibrin reaction.

Let's use the definition of the hydrodynamic volume as a volume of sphere with diameter being equal to the largest diameter of a molecule. For example, for a rod like molecule such sphere has a diameter equal to the molecule length. Semi-dilute condition for oligomers $c_{12}$ will be fulfilled when relative hydrodynamic volume is equal to unity:

$$
v_{e}=\frac{4}{3} \pi\left(\frac{L_{12}}{2}\right)^{3} c_{12 \mathrm{thr}} N_{A}=1
$$

where $c_{12 \text { thr }}$ is a threshold concentration for oligomers of size 12, $N_{A}$ is Avogadro number. So it can be estimated $c_{12}$ :

$$
c_{12 \mathrm{thr}}=\frac{3}{4 \pi N_{A}}\left(\frac{L_{12}}{2}\right)^{-3} \text {. }
$$

Second assumption of the model: the semi-dilute solution of rod-like oligomers $c_{12}$ polymerize with a creation of a gel nucleus which later absorbs rest of $c_{12}$ oligomers. Fresh gel polymer threads are thin, or to put it in other words, threads do not contain many lateral aggregates.

So, to describe behavior as fast, virtually instantaneous, transition from free $c_{12}$ to gel after a threshold achievement, an appropriate term should be added:

$$
\frac{d c_{12}}{d t}=D_{12} \Delta c_{12}+k_{\text {poly }} c_{1} c_{11}-\frac{c_{12}}{\varepsilon}\left(\frac{\left(c_{12} / c_{12 \mathrm{thr}}-1\right)+\sqrt{\left(c_{12} / c_{12 \mathrm{thr}}-1\right)^{2}}}{\left(c_{12} / c_{12 \mathrm{thr}}-1\right)+\sqrt{\left(c_{12} / c_{12 \mathrm{thr}}-1\right)^{2}}+\delta}\right)^{\gamma} .
$$

The term in round brackets describes a threshold polymerization function. Fast turning on of this function leads to a gel formation in the space given. Parameter $\varepsilon$ describes characteristic times of polymerization. It is supposed that $\varepsilon$ is a small value. Parameter $\gamma$ is responsible for the speed of polymerization reactions switching on. Parameter $\delta$ is a small positive value, preventing denominator to become zero. It has a meaning of machine epsilon in programming. Authors presume the first order kinetics of this term based on the following consideration: after gel nucleus has been formed in the current macro point then the gel growth speed will be proportional to the $c_{12}$ concentration. The formation of nucleus in semi-dilute solution is determined by another, faster dependency. So, the rate limiting stage will be of first order on $c_{12}$.

Basing on estimation for $c_{12 \text { thr }}$ let's assess polymer threads length $l_{0}$ in a unit volume of nucleus under condition of $v_{e}=1$ (in the moment of gelation):

$$
l_{0}=\alpha N_{A} c_{12 \mathrm{thr}}\left(L_{12}\right) L_{12}=\frac{3 \alpha}{2 \pi}\left(\frac{L_{12}}{2}\right)^{-2} .
$$

Below such a mesh will be referred to as a "young". 
For the thread length evolution equation so far we can write:

$$
\frac{d l}{d t}=\alpha N_{A} L_{12} \frac{c_{12}}{\varepsilon}\left(\frac{\left(c_{12} / c_{12 \mathrm{thr}}-1\right)+\sqrt{\left(c_{12} / c_{12 \mathrm{thr}}-1\right)^{2}}}{\left(c_{12} / c_{12 \mathrm{thr}}-1\right)+\sqrt{\left(c_{12} / c_{12 \mathrm{thr}}-1\right)^{2}}+\delta}\right)^{\gamma} .
$$

The $c_{12}$ oligomers aggregation process has not been thoroughly studied yet. Supposedly, it is driven by Van der Waals forces. Nevertheless, this process plays a key role in the fibrin-polymer gelation. Authors propose to split it in two subprocesses. The first subprocess is a paired association of $c_{12}$ oligomers that forms "elongated" aggregate with a size $2 \alpha L_{12}(1 / 2<\alpha<1)$. So, the oligomers join with a certain overlapping. Second subprocess is a lateral aggregation of $c_{12}$ oligomer on the existing mesh fiber or freely floating aggregate (yet even such an aggregate consists only of two associated $c_{12}$ oligomers). We suppose that lateral association do not elongate an aggregate.

Let constant $k_{\text {lat }}$ characterizes the process of lateral aggregation of $c_{12}$ oligomers on polymer with a thread specific length $l$. And constant $k_{\text {elong }}$ characterizes the interaction of two oligomers with a formation of a new fiber (thread) of length $2 \alpha L_{12}$. Then it can be written as:

$$
\begin{aligned}
\frac{\partial c_{12}}{\partial t}= & D_{12} \Delta c_{12}+k_{\text {poly }} c_{11} c_{1}-2 k_{\text {elong }} c_{12}^{2}-k_{\text {lat }} \frac{l}{L_{12}} c_{12} \\
& -\frac{c_{12}}{\varepsilon}\left(\frac{\left(c_{12} / c_{12 \mathrm{thr}}-1\right)+\sqrt{\left(c_{12} / c_{12 \mathrm{thr}}-1\right)^{2}}}{\left(c_{12} / c_{12 \mathrm{thr}}-1\right)+\sqrt{\left(c_{12} / c_{12 \mathrm{thr}}-1\right)^{2}}+\delta}\right)^{\gamma} .
\end{aligned}
$$

The equation describing thread length evolution changes as follows:

$$
\frac{\partial l}{\partial t}=2 \alpha N_{A} L_{12} k_{\text {elong }} c_{12}^{2}+\alpha N_{A} L_{12} \frac{c_{12}}{\varepsilon}\left(\frac{\left(c_{12} / c_{12 \mathrm{thr}}-1\right)+\sqrt{\left(c_{12} / c_{12 \mathrm{thr}}-1\right)^{2}}}{\left(c_{12} / c_{12 \mathrm{thr}}-1\right)+\sqrt{\left(c_{12} / c_{12 \mathrm{thr}}-1\right)^{2}}+\delta}\right)^{\gamma} .
$$

Summarizing the above said, it should be kept in mind, that the processes of the lateral aggregation and the elongation can take place in the solution lower than the concentration of $c_{12}$ oligomers achieve threshold and gel formed. Inside of the gel elongation process results in the polymer threads length increase as model is not accounting for free floating aggregates inside of the gel. All "free floating" polymer aggregates formed are considered to build in into polymer gel during gelation process. As a size of the polymer aggregates is rather large (around $0.5-1 \mu \mathrm{m}$ ), we will neglect their passive diffusion (model assumption 3). Then, after the gel formation all surfaces where polymer thread has length of $l_{0}$ per unit volume can be considered as a phase border.

At this point it is worth to mention that the polymer aggregates, let alone the gel, are considered as virtually inaccessible for fibrin monomer attachment. Neighboring $c_{12}$ threads in aggregate can be regarded as steric obstacles for fibrin monomers to align with open $c_{12}$ ends. The exact measure of this hindrance is of course to be verified by experiment, but it should be very significant just only from geometrical considerations.

\subsection{Mesh}

Let's consider the next stage of fibrin-polymer gel formation. Suppose the nucleus of the gel has already been formed. 
Here we introduce the 4th assumption of the model: let's assume that a cell diameter $d_{0}$ for the young fibrin-polymer mesh is a process intrinsic property. We assume that $d_{0}$ is not dependent on concentrations, say $c_{12}$, but depends on the fibrin(ogen) form properties in consideration (normal or mutant) and, may be, on the lateral and elongation constants ratio. In the course of its evolution, the mesh can become denser, new fibers will appear and $d$ decrease.

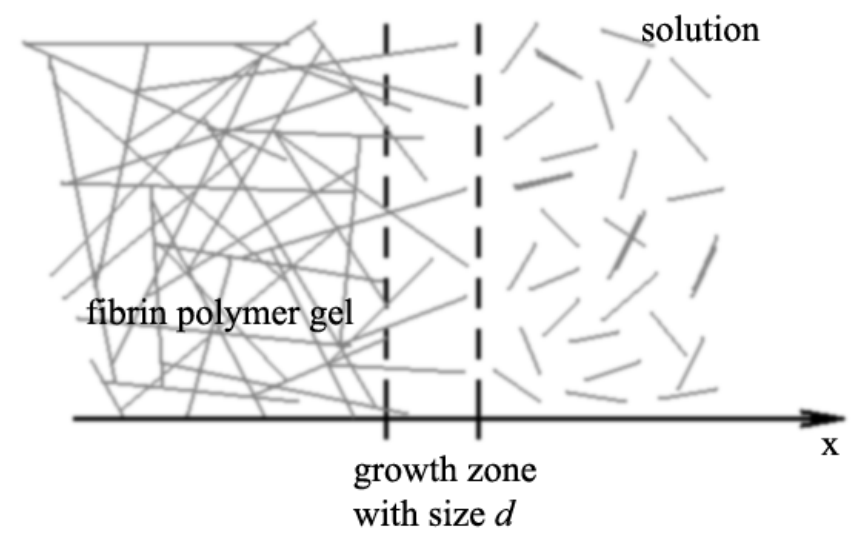

Figure 3: Transitional or mesh border zone under magnification. Fibrin polymer gel is on the left. Non gelled solution of aggregates and $c_{12}$ oligomers are on the right. The transitional zone size approximately corresponds to the pore diameter in a young gel $\left(d_{0}\right)$. The presence of active sites, capable to catch $c_{12}$ oligomers in great numbers is a specific feature of a transitional zone.

Suppose the size of transitional zone (see Fig. 3) is much smaller than entire modelling volume space dimension and it is rich in sites capable to join $c_{12}$ oligomers (5th assumption). The basis for such an assumption is the following. When polymer mesh appears from a semi dilute solution of rod-like $c_{12}$ oligomers, such young mesh will contain large number of sites yet another $c_{12}$ oligomer can join. Due to Brownian motion the young mesh will reorder by aggregation of nearby $c_{12}$ "open" ends. This reordering process characteristic time is of the same magnitude as time of $c_{12}$ oligomer drift on distance $d_{0}$. In the model we do not consider reordering by itself and the decrease of $l$ in the reordered volume. But disappearance of $c_{12}$ "open" ends is accounted for. So, the gel-solution border can be considered as a discontinuity surface.

Outside of discontinuity for $c_{12}$ oligomer it can be written:

$$
\begin{aligned}
\frac{\partial c_{12}}{\partial t}=-\frac{\partial W}{\partial x}+[ & k_{\text {poly }} c_{11} c_{1}-2 k_{\text {elong }} c_{12}^{2}-k_{\text {lat }} \frac{l c_{12}}{L_{12}}- \\
& \left.-\frac{c_{12}}{\varepsilon}\left(\frac{\left(c_{12} / c_{12 \mathrm{thr}}-1\right)+\sqrt{\left(c_{12} / c_{12 \mathrm{thr}}-1\right)^{2}}}{\left(c_{12} / c_{12 \mathrm{thr}}-1\right)+\sqrt{\left(c_{12} / c_{12 \mathrm{thr}}-1\right)^{2}}+\delta}\right)^{\gamma}\right] .
\end{aligned}
$$

In the last equation the diffusion driven flow $W$ was introduced. The 6th assumption of the model is that fibrin polymer mesh (even young) doesn't influence passive diffusion of oligomers up to 
size 12. So, for the oligomer diffusion Fick's law can be used:

$$
W=-D_{12} \frac{\partial c_{12}}{\partial x}
$$

For simplicity an elementary volume $d \Omega=S \delta x$ with a unity surface $S$ can be introduced. Let the border sol-gel transition wave be located inside the mentioned volume. Wave passes $V \delta t$ for a elementary time $\delta t$, where $V$ is a wave's speed. As $\delta t$ is elementary, it can be counted that $V$ is a constant. Without loss of generality the wave is assumed to be propagating from the left to right. Let consider the evolution of $c_{12}$ in the volume in hand. Now we account not only for volume reactions but also for diffusion flows through the primary volume borders. According to the 5th assumption we also account for reactions related to oligomers joining $c_{12}$ to newly formed young fibrin gel polymer threads on the phase border. Than the following balance equation can be written as:

$$
\begin{aligned}
\left(c_{12}(t+\delta t)-c_{12}(t)\right) \delta x=( & -W(x+\delta x)+W(x)) \delta t+k_{\text {surf }} c_{12}(\theta(x-V \delta t)-\theta(x)) \delta t+ \\
+ & {\left[k_{\text {poly }} c_{11} c_{1}-2 k_{\text {elong }} c_{12}^{2}-k_{\text {lat }} \frac{l c_{12}}{L_{12}}-\right.} \\
& \left.-\frac{c_{12}}{\varepsilon}\left(\frac{\left(c_{12} / c_{12 \mathrm{thr}}-1\right)+\sqrt{\left(c_{12} / c_{12 \mathrm{thr}}-1\right)^{2}}}{\left(c_{12} / c_{12 \mathrm{thr}}-1\right)+\sqrt{\left(c_{12} / c_{12 \mathrm{thr}}-1\right)^{2}}+\delta}\right)^{\gamma}\right] \delta t \delta x,
\end{aligned}
$$

where constant $k_{\text {surf }}$ describes the surface reaction speed of oligomers $c_{12}$ joining in the transition zone on the phase (gel-sol) border.

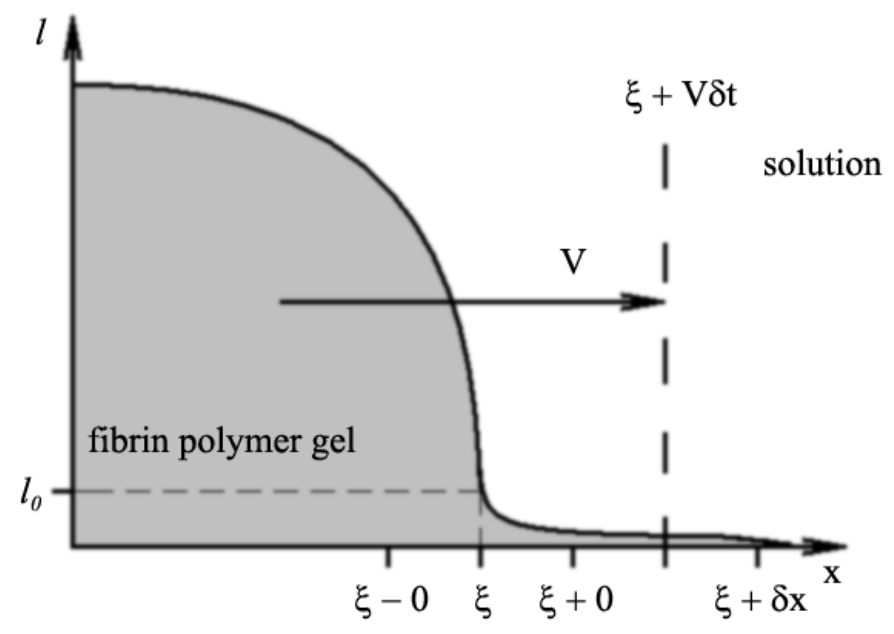

Figure 4: Qualitative dependence of fibrin-polymer threads length from coordinate. Gelated volume border is moving from left to right with a speed $V$. The gelation criteria is an achievement of threshold value $l_{0}$ by length of fibrin-polymer threads in the unit volume. 
Dividing both sides by $\delta t \delta x$ and passing to the limit the equation for $c_{12}$ concentration in a gel border vicinity can be derived:

$$
\begin{aligned}
\frac{\partial c_{12}}{\partial t}=-\frac{\partial W}{\partial x}+[ & k_{\text {poly }} c_{11} c_{1}-2 k_{\text {elong }} c_{12}^{2}-k_{\text {lat }} \frac{l c_{12}}{L_{12}}- \\
& \left.-\frac{c_{12}}{\varepsilon}\left(\frac{\left(c_{12} / c_{12 \mathrm{thr}}-1\right)+\sqrt{\left(c_{12} / c_{12 \mathrm{thr}}-1\right)^{2}}}{\left(c_{12} / c_{12 \mathrm{thr}}-1\right)+\sqrt{\left(c_{12} / c_{12 \mathrm{thr}}-1\right)^{2}}+\delta}\right)^{\gamma}\right],
\end{aligned}
$$

where $V$ is gelation wave front speed (see Fig. 4). If according to the 6th assumption Fick's law is applicable for description of passive diffusion inside and outside of gel then for discontinuity of $c_{12}$ diffusion flow a condition is obtained:

$$
\begin{gathered}
D_{12}\left\{\frac{\partial c_{12}(x+0)}{\partial x}-\frac{\partial c_{12}(x-0)}{\partial x}\right\}=k_{\text {surf }} c_{12}, \\
{\left[-D_{12} \frac{\partial c_{12}}{\partial x}\right]=-k_{\text {surf }} c_{12} .}
\end{gathered}
$$

In a similar way there can be derived for the specific length of fibrin-polymer threads in the unit volume:

$$
\frac{\partial l}{\partial t}=\alpha L_{12} k_{\text {elong }} c_{12}^{2}+\alpha L_{12} \frac{c_{12}}{\varepsilon}\left(\frac{\left(c_{12} / c_{12 \mathrm{thr}}-1\right)+\sqrt{\left(c_{12} / c_{12 \mathrm{thr}}-1\right)^{2}}}{\left(c_{12} / c_{12 \mathrm{thr}}-1\right)+\sqrt{\left(c_{12} / c_{12 \mathrm{thr}}-1\right)^{2}}+\delta}\right)^{\gamma}
$$

and equation for the magnitude of discontinuity $[l V]=-\alpha k_{\text {surf }} c_{12}$. Based on the conclusion from the 2 nd assumption regarding the fixed fibrin-polymer thread length of young gel the latter equation can be rewritten as:

$$
V\left(l(\xi+0)-l_{0}\right)=-\alpha k_{\text {surf }} c_{12}(\xi) .
$$

So, the gelation wave speed could be estimated as:

$$
V=-\frac{\alpha k_{\text {surf }} c_{12}(\xi)}{l(\xi+0)-l_{0}}
$$

An important measurable value in clotting experiments is a fibrin polymer total mass. In the proposed model a fibrin polymer specific mass can be equated with a mass of $c_{12}$ aggregates including mesh itself. So, protein mass density away from the gel border can be described as:

$$
\frac{\partial \rho}{\partial t}=m_{12}\left[2 k_{\text {elong }} c_{12}^{2}+k_{\text {lat }} \frac{l c_{12}}{L_{12}}-\frac{c_{12}}{\varepsilon}\left(\frac{\left(c_{12} / c_{12 \mathrm{thr}}-1\right)+\sqrt{\left(c_{12} / c_{12 \mathrm{thr}}-1\right)^{2}}}{\left(c_{12} / c_{12 \mathrm{thr}}-1\right)+\sqrt{\left(c_{12} / c_{12 \mathrm{thr}}-1\right)^{2}}+\delta}\right)^{\gamma}\right] .
$$

The mass density discontinuity magnitude on the phase border can be derived from balance relations:

$$
-V(\rho(\xi+0)-\rho(\xi-0))=m_{12} k_{\text {surf }} c_{12},
$$

where $m_{12}$ is the mass of one $c_{12}$ oligomer.

So, herein the closed system of equations for the phase transition wave propagation description is demonstrated. 


\section{Discussion}

Utilizing equation (2.1) the value of $c_{12 \mathrm{thr}} \approx 1.23 \cdot 10^{-7} \mathrm{M}$ can be computed. It corresponds to $1.46 \cdot 10^{-6} \mathrm{M}$ of monomeric fibrin incorporated in the oligomers of length 12 . Upper physiological norm of fibrinogen is around $1.2 \cdot 10^{-5} \mathrm{M}$ or $4 \mathrm{mg} / \mathrm{ml}$. As an indirect supporting evidence for this estimation experimental results from [18] can be cited. It has been proved in [18] that the minimum fibrinogen level which is necessary for appearance of the gel in $100 \mathrm{~s}$ is in range from $0.9 \cdot 10^{-6}$ to $2 \cdot 10^{-6} \mathrm{M}$ under thrombin surplus conditions.

It is useful to consider the relation between $l_{0}$ and pore diameter $d_{0}$. The cubic-alike mesh can be taken as a simplified model. The sum of cubic cell edges length is equal to $12 d$, volume is- $d^{3}$. Each edge of the cubic cell belongs to 4 neighbors. Thus, the relation between $l_{0}$ and pore diameter $d_{0}$ is equal to $l_{0}=3 / d_{0}^{2}$.

Estimation for the polymer thread specific length for young gel according to (2.2) yields $l_{0}=$ $\alpha \cdot 2.2 \cdot 10^{9} \mathrm{~cm}^{-2}$. Correspondingly, the estimation for $d_{0}$ yields $d_{0}=369 / \sqrt{\alpha} \mathrm{nm}$. If it is supposed that $\alpha=0.75$, then the value $l_{0}$ is approximately 10 times higher, and $d_{0}$ is approximately 3 times lower than the data known from experiments for mature fibrin meshes. Such deviation could be explained by the fact, that proposed model do not account for clot maturation and mesh reordering. Unfortunately, there is no direct experimental technique available now to prevent fibrin mesh from growing elder, so there is no data regarding young meshes so far.

An important and relatively easy measurable value in the clotting experiments is a fibrin polymer mass. Also in the direct experiments it is possible to determine fibrin mesh permeability, which depends on the polymer thread specific length. Proposed model predicts these values directly. In the same time the current model can't predict viscous and elastic properties of the fibrin-polymer mesh as it does not take into account junction points quality and morphology.

The model in hand is important for the hydrodynamics as it allows finding the clot border and defines areas of non-restricted flow outside of the mesh and filtration flow inside of the gel well. This, in turn, allows setting correctly the border conditions on sol-gel boundaries.

One of the base assumptions of model infers the existence of additional mechanism of gel growth, that is joining of $c_{12}$ oligomers to transitional zone on the gel border. As this mechanism has not been mentioned before its overall contribution is yet to be experimentally verified.

Also it is necessary to work on specification of threshold function which turns on gelation when $c_{12}$ concentration achieves $c_{12 \text { thr }}$ and solution becomes semi dilute. An important property of the proposed model is a capability to take into account an influence on polymerization of such a mechanical factor as shear rate. The criteria of semi dilute solution will shift considerably in shear flow. This means that flow influence on fibrin polymerization and gelation can be considered without changes in model structure. In case of shear flow presence relative hydrodynamic volume for $c_{12 \text { thr }}$ can be written as:

$$
v_{h}=\frac{4}{3} \pi N_{A}\left(\frac{L_{12}}{2}\right) c_{12 \operatorname{thr}}\left(\frac{L_{12}}{2}-\xi \sqrt{I_{2}(\dot{\gamma})}\right)^{2}=1,
$$

where $I_{2}(\dot{\gamma})$ is a quadratic invariant on the strain velocity tensor, $\xi$ characterizes shear influence on the eccentricity of perpendicular to flow axis of elliptical body describing hydrodynamic volume of oligomer $c_{12 \mathrm{thr}}$. 


\section{Conclusion}

The current paper presents an approach to the sol-gel transition description while fibrin polymerization is similar to the generalized Stefan problem on the phase transition border motion. The specificity of the approach proposed is that flow discontinuity is raised due to the reactions on the border, not due to a difference of diffusion or thermal conductivity coefficients. Unlike, in case of the classic Stefan problem [23] the discontinuity magnitude is not constant but depends on the oligomer concentration. Note, that this feature is a direct consequence of an inference about the existence of transitional zone on the border sol-gel. The proposed model can be modified to account for shear flow influence on fibrin polymerization and gel transition.

\section{Acknowledgements}

Authors are grateful to Dr. V.N. Buravtsev (IChPh RAS), Dr. Prof. F.I. Ataullakhanov (HRC RAMS), Dr. M.A. Panteleev (HRC RAMS), Dr. E.I. Sinauridze (HRC RAMS), M.V. Petrukhno (MIPT) for consulting and discussions.

This work has been supported by the 14th Program of the Presidium of Russian Academy of Science, Project No. 2010-111-RICCR.

\section{References}

[1] World health report 2004 statistical annex [Electronic resource]: Annex Table 2: Deaths by cause, sex and mortality stratum in regions, estimates for 2002. World Health Organization. http://www.who.int/whr/2004/annex/en/index.html.

[2] M. Anand, K. Rajagopal, K.R. Rajagopal A model incorporating some of the mechanical and biochemical factors underlying clot formation and dissolution in flowing blood. J. Theor. Med. 5 (2003), 183-218.

[3] F.I. Ataullakhanov, G.T. Guriia, A.Iu. Safroshkina Spatial aspects of the dynamics of blood coagulation. II. Phenomenological model. Biofizika, 39 (1994) 97-104 (in Russian).

[4] F.I. Ataullakhanov, V.I. Zarnitsina, et al. Spatio-temporal dynamics of blood coagulation and pattern formation. A theoretical approach. Int. J. Bifurc. Chaos, 12 (2002), No. 9, 1985-2002.

[5] F.I. Ataullakhanov, G.T. Guriia. Spatial aspects of the dynamics of blood coagulation. I. Hypothesis Biofizika, 39 (1994), 89-96 (in Russian).

[6] F.I. Ataullakhanov, R.I. Volkova, et al. Spatial aspects of blood coagulation dynamics. III. Growth of clots in vitro. Biofizika, 40 (1995) 1320-1328 (in Russian).

[7] B. Blomback, K. Carlsson, et al. Fibrin in human plasma: gel architectures governed by rate and nature of fibrinogen activation. Thromb Res., 75 (1994), No. 5, 521-538. 
[8] M.E. Carr Jr., C.L. Hardin. Fibrin has larger pores when formed in the presence of erythrocytes Amer. J. Physiol., 253 (1987), No. 2, 1069-1073.

[9] M.E. Carr Jr, J. Hermans. Size and density of fibrin fibers from turbidity. Macromolecules, 11 (1978), No. 1, 46-50.

[10] C.E. Dempfle, P.N. Knoebl. Blood coagulation and inflammation in critical illness the importance of the protein C pathway. UNI-MED, Bremen, 2008.

[11] S.L. Diamond. Engineering design of optimal strategies for blood clot dissolution. Ann. Rev. Biomed. Engrg, 1 (1999) 427-461.

[12] M. Doi, S.F. Edwards. Theory of polymer dynamics. Acad. Press, New York, 1986.

[13] E.A. Ermakova, M.A. Panteleev, E.E. Shnol. Blood coagulation and propagation of autowaves in flow. Pathophysiol. Haemost. Thromb., 34 (2005), No. 2-3, 135-142.

[14] P.-G. de Gennes. Scaling concepts in polymer physics. Cornell, London, 1979.

[15] R.R. Hantgan, J. Hermans. Assembly of fibrin. A light scattering study. J. Biol. Chem., 254 (1979) No. 22, 11272-11281.

[16] R. Kita, A. Takahashi, et al. Formation of fibrin gel in fibrinogen-thrombin system: static and dynamic light scattering study. Biomacromolecules, 3 (2002), No. 5, 1013-1020.

[17] R. Marchi, M. Meyer, et al. Biophysical characterization of fibrinogen Caracas I with an Aalpha-chain truncation at Aalpha-466 Ser: identification of the mutation and biophysical characterization of properties of clots from plasma and purified fibrinogen Blood Coagul. Fibrinolys., 15 (2004), No. 4, 285-293.

[18] G. Marx. Simulating fibrin clotting time. Med. Biol. Engrg. Comput., 44 (2006), 79-85.

[19] Medved' L, Ugarova T, et al. Electron microscope investigation of the early stages of fibrin assembly. Twisted protofibrils and fibers J. Mol. Biol., 216 (1990), No. 3, 503-509.

[20] M.W. Mosesson, J.P. DiOrio, et al. Evidence for a second type of fibril branch point in fibrin polymer networks, the trimolecular junction Blood, 82 (1993), No. 5, 1517-1521.

[21] M.W. Mosesson. Fibrinogen and fibrin structure and functions. J. Thromb. Haemost., 3 (2005), No. 8, 1894-1904.

[22] M.A. Panteleev, M.V. Ovanesov, et al. Spatial propagation and localization of blood coagulation are regulated by intrinsic and protein C pathways, respectively. Biophys. J. 90 (2006), No. 5, 1489-1500.

[23] G.G. Tsipkin. Flows with phase transitions in porous media. Fizmatlit, Moscow, 2009 (in Russian). 
[24] J.W. Weisel. Fibrinogen and fibrin. Adv. Protein Chem., 70 (2005), 247-299.

[25] J.W. Weisel, C. Nagaswami, L. Makowski. Twisting of fibrin fibers limits their radial growth. Proc. Nat. Acad. Sci. USA, 84 (1987), No. 24, 8991-8995.

[26] D.M. Zubairov. Molecular basis of clotting and thrombus formation. Fen Press, Kazan, 2000 (in Russian). 